\title{
Fading Characteristics of Wireless Channel on High-Speed Railway in Hilly Terrain Scenario
}

\author{
Fengyu Luan, Yan Zhang, Limin Xiao, Chunhui Zhou, and Shidong Zhou \\ State Key Laboratory on Microwave and Digital Communications, Tsinghua National Laboratory for Information \\ Science and Technology, Department of Electronic Engineering, Tsinghua University, Beijing 100084, China
}

Correspondence should be addressed to Yan Zhang; yanz@tsinghua.edu.cn

Received 13 July 2012; Revised 26 January 2013; Accepted 28 January 2013

Academic Editor: Cheng-Xiang Wang

Copyright (C) 2013 Fengyu Luan et al. This is an open access article distributed under the Creative Commons Attribution License, which permits unrestricted use, distribution, and reproduction in any medium, provided the original work is properly cited.

This paper focuses on the fading characteristics of wireless channel on High-Speed Railway (HSR) in hilly terrain scenario. Due to the rapid speed, the fading characteristics of HSR channel are highly correlated with time or Transmit-Receive distance and have their own special property. To investigate the fading characteristics, the measurement is conducted on the Guangzhou-Shenzhen passenger-dedicated line in China with the speed of $295 \mathrm{~km} / \mathrm{h}$ in the data-collection area at $2.4 \mathrm{GHz}$. From the measured data, the amplitude of each path is estimated by using the Subspace-Alternating Generalized Expectation-Maximization (SAGE) algorithm along with other parameters of channel impulse responses. Then the fading parameters, including path loss, shadow fading, and $K$-factor, are analysed. With the numerical results in the measurement and analysis, the fading characteristics have been revealed and modelled. It is supposed that this work has a promotion for HSR communication system design and improvement.

\section{Introduction}

High-Speed Railway (HSR) has the properties of large conveying capacity, good security, low-energy consumption, and vast economic benefits $[1,2]$. With rail speed designed to travel at no less than $200 \mathrm{~km} / \mathrm{h}$ [1], HSRs are widely deployed around the world. For example, the Japanese Shinkansen started in the year of 1963, at a maximum speed of $320 \mathrm{~km} / \mathrm{h}$, while the Eurostar is running across the European continent with the speed reaching $300 \mathrm{~km} / \mathrm{h}$. In recent years, China has paid more attention to the HSR and has made great progress. Upon the end of year 2011, the operating mileages surpassed 1300 kilometers, making HSR an increasingly important form of transportation.

However, there is a great difference for wireless channel between the HSR scenario and the typical scenario, especially in fading characteristics. In general, communication systems are very sensitive to the fading of the wireless channel, which causes intercarrier interfaces and degrades system performance [3]. The HSR scenario has the special property of high velocity and rapidly changing environment. So the channel exhibits great position-based fading characteristics, which introduces problems to increase the wireless communication capacity and improve the wireless communication quality. On the other hand, the fading characteristics play an important role in system simulation. Only the influence of fast small-scale fading is considered in most communication simulation methods. But in HSR scenario, path loss and shadow fading are also changed in a continuous simulation process with a long distance caused by high moving speed. For these reasons, having a good knowledge of the HSR wireless channel fading characteristics will help to give a reference for the HSR communication system simulation.

It is significant to get reliable and realistic fading characteristics of the HSR wireless channel through the wideband channel measurements. The HSR scenarios can be divided into different types, including hilly terrain, viaduct, tunnel, cutting, and station. Several HSR channel measurements have been performed by researchers worldwide. The viaduct, tunnel, cutting, and hilly terrain scenarios are measured and modeled, respectively. Liu et al. give the position-based measurement result of HSR channel fading parameters at $2.35 \mathrm{GHz}$ in viaduct scenario [4]. The measurements of path loss and delay spread in tunnels of different sizes at $2.154 \mathrm{GHz}$ 
are performed by Helsinki University of Technology [5]. Cichon et al. also build up a ray-optical propagation model for tunnel channel [6]. The Rice $K$-factor in cutting scenario is presented in [7] with the measurement frequency of 930.2 MHz. Some channel parameters including delay spread and $K$-factor in different scenarios and different railways are compared [8]. The nonstationary MIMO channel model based on the space-time-frequency correlation function for HSR communication system is proposed for modeling the time-variant property of different taps [9].

To the best of our knowledge, existing researches seldom pay attention to the position-based property of the hilly terrain HSR channel. The hilly terrain occupies $69.1 \%$ of the area in China. And the newly built lines of China, such as the Chengdu-Guiyang passenger-dedicated line and Guangzhou-Shenzhen passenger-dedicated line, run through vast area of hilly terrain. The HSR hilly terrain channel measurement and modeling are of great significance. Compared with other scenarios, the scattering and position-based property of the multipath propagation is more complicated and more obvious in hilly terrain. Both large-scale fading and small-scale fading are changed with the train fast moving. In this paper, the fading characteristics of the wireless channel in hilly terrain scenarios are analyzed from the actual channel measurement data. We also attempt to give out the models which describe the position-based statistic property of different channel parameters which reveal the characteristics, such as path loss, shadow fading, and $K$-factor. The reliable and realistic model serves as the foundation for designing and improving the HSR communication systems.

The main contributions of our works consist of the measurement newly conducted in the HSR hilly terrain scenario, the proposition of the position-based models of different channel parameters, and the application of the SubspaceAlternating Generalized Expectation-Maximization (SAGE) algorithm. SAGE algorithm is regarded as a high-resolution channel parameter estimation method and fits for dealing with the several position-based channel parameters by jointing the time and frequency domains. The properties of path loss, the lognormal shadowing, and $K$-factor are concluded in this paper. These properties are compared with the current models and explained in detail by investigating the TransmitReceive (T-R) distance and actual measurement scenario.

The remainder of the paper is organized as follows. In Section 2, the description of hilly terrain scenario and the arrangement of the measurement are outlined. And in Section 3, the details of the SAGE algorithm application are laid out. In Section 4, statistic results of position-based channel parameters which are used for building the channel fading models are discussed and analyzed. The paper is concluded in Section 5.

\section{Measurement Setup}

The measurement in this paper is taken on the GuangzhouShenzhen passenger-dedicated line. The main terrains along the railway are hilly terrain and plain with viaducts. These two types of scenarios are almost distributed alternatively.
Here the paper focuses on the hilly terrain scenario. Figure 1 shows satellite map of our measured hilly terrain scenario. It is generated using the Google Maps tool [10]. The white line is referred to as the railway from West to East. And the base station is located at the position which is $63 \mathrm{~km}$ from Guangzhou and $41 \mathrm{~km}$ from Shenzhen as the green arrow shows along the railway with the GPS coordinates of $22.830558 \mathrm{~N}, 113.860235 \mathrm{E}$. In the data-collection area, many kinds of scattering objects are observed around the railway at the foot of hills. Besides hills, there is a big pond near the railway in the distance of about $600 \mathrm{~m}$ from the base station. The areas with an abundance of buildings, factories, and warehouses appear in the distance of about $800 \mathrm{~m}$ and $2200 \mathrm{~m}$, respectively. It can be seen that an expressway intersects with the railway from far to near. In this scenario, the relative altitudes of these main scattering objects are mostly below $200 \mathrm{~m}$. In summary, there are several significant properties which have huge effect on the channel fading characteristics in the HSR hilly terrain scenario during the signal transmission.

(1) With the high-altitude transmit antenna and lowaltitude obstacles, the Line of Sight (LOS) is observable and can be detected along the entire railway.

(2) In hilly terrain, the propagation environment is high density scattered, and scattering objects are distributed irregularly and nonuniformly. The constructive and destructive interference among different multipath components (MPCs) with similar delay is serious. This phenomenon has influence on the channel fading characteristics.

(3) The high speed will not only cause the large Doppler frequency shift, but also bring the rapid change to the channel parameters. When the train moves quickly, both the propagation environment and the angles of incidence vary quickly. The signal will go through different propagation paths. At the same time, the channel parameters are changed.

Our measurement is conducted by using the THU channel sounder. The parameters of the measurement setup are listed in Table 1 . The center frequency is $2.4 \mathrm{GHz}$, and the bandwidth is $40 \mathrm{MHz}$. The transmit antenna is mounted on the base station tower (Figure 2(a)). It is a directional antenna pointing at the direction of train travelling with the gain of $17 \mathrm{dBi}$. The receive antenna is fixed on the window of the high-speed moving train carriage (Figure 2(b)). It is vertically polarized and omnidirectional with the gain of $7 \mathrm{dBi}$. The transmitter and the receiver are both locked to GPS-derived $10 \mathrm{MHz}$ references, thereby eliminating frequency offsets between the two time bases. In the transmitter, the test signal is fed through the power amplifier to the transmit antenna with the total power of $20 \mathrm{dBm}$. After receiving the test signal, the receiver does the low-noise amplification and down conversation. Then the signal is sampled with the sample rate of $100 \mathrm{MHz}$ and stored to the disk array of a server for offline analysis [11].

The test signal is regarded as a linear frequency modulated (LFM) sequence. Its length is $12.8 \mu \mathrm{s}$. Due to the good 


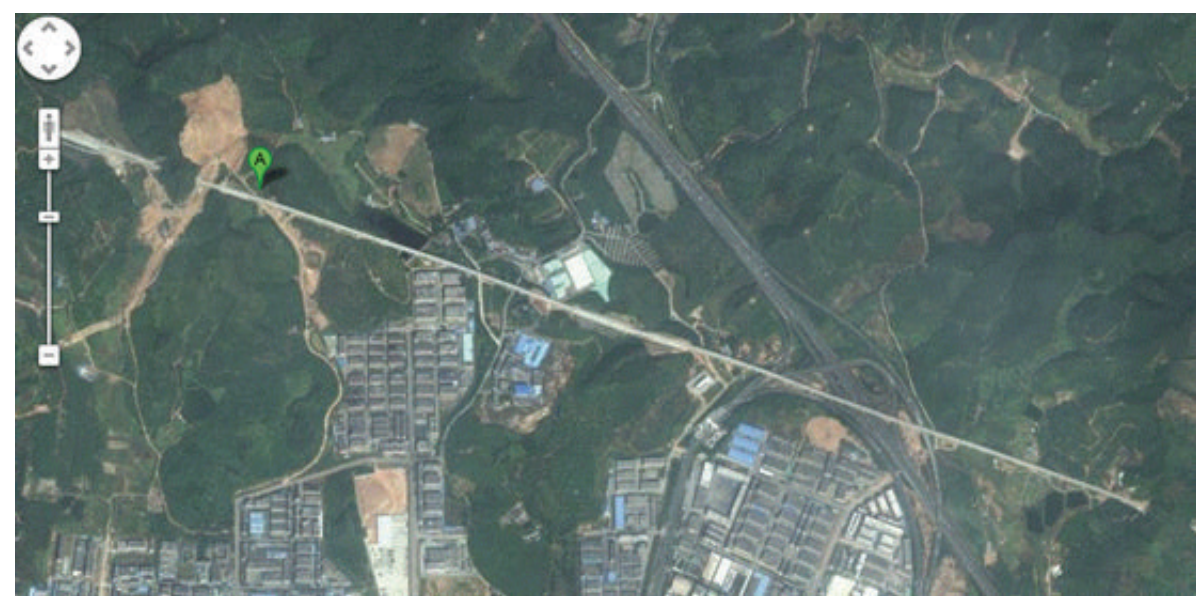

FIGURE 1: Satellite map of measured hilly terrain area.

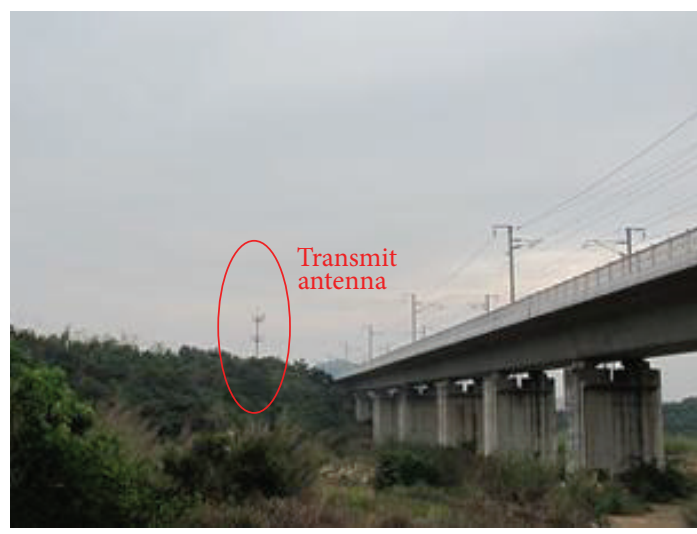

(a)

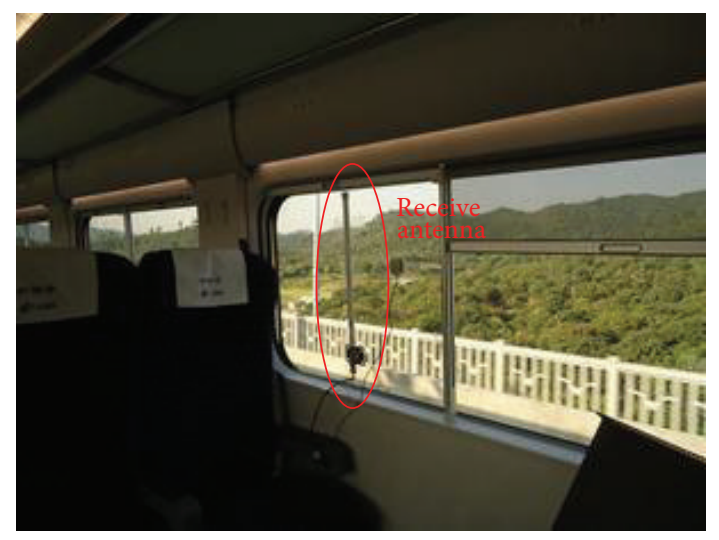

(b)

FIgURE 2: (a), (b) are photos of the propagation environments at the transmit side and receive side, respectively.

TABLE 1: Parameters of the measurement setup.

\begin{tabular}{lc}
\hline Parameter & Value \\
\hline Central frequency & $2.4 \mathrm{GHz}$ \\
Bandwidth & $40 \mathrm{MHz}$, \\
Transmitter power & $20 \mathrm{dBm}$ \\
& $1 \mathrm{Tx}$ and $1 \mathrm{Rx}$ \\
Antenna pattern and gain & Tx: directional antenna \\
& with $17 \mathrm{dBi}$ \\
& Rx: Onmidirectional \\
& antenna with $7 \mathrm{dBi}$ \\
Tx height & $30 \mathrm{~m}$ \\
Rx height & $1.2 \mathrm{~m}$ \\
Test signal length & $12.8 \mu \mathrm{s}$ \\
The minimum distance between Tx & $10 \mathrm{~m}$ \\
and railway & $12.4 \mathrm{~dB}$ \\
The penetration loss of the window & $295 \mathrm{~km} / \mathrm{h}$ \\
The speed of HSR train &
\end{tabular}

autocorrelation property of the LFM sequences, the measurement principle of LFM signal is that the received sequence is correlated with the local copy of the sequence at the receiver to produce the measured channel impulse response. Then the offline and broadband features simplify the system operation. After the measurement data is stored in the server, the channel parameter estimation method such as SAGE algorithm is utilized to search and separate the different multipath components, according to the different delay and Doppler frequency of each component. Furthermore, the calibrations of the response of radio frequency (RF) at both Tx side and Rx side and the measurement of the penetration loss of the carriage window are also conducted in each measurement.

In the measured hilly terrain, the height of transmit antenna $h_{b}$ is $30 \mathrm{~m}$, and the height of receive antenna $h_{m}$ is $1.2 \mathrm{~m}$. The minimum distance between transmit antenna and the railway is $10 \mathrm{~m}$. The GPS system of the train shows the speed of the train in the data-collection area remains $295 \mathrm{~km} / \mathrm{h}$. The total length of the measured route is $2600 \mathrm{~m}$ from the base station. Beyond this distance, the train will rush into a tunnel. The main reflections of the electromagnetic wave come from ground, hill, buildings, ponds, and plantings as mentioned above.

Providing the wireless signal coverage for HSR is a very important and tremendous task, which is related to both the 
channel fading characteristics and coverage scheme. There are two schemes for HSR wireless signal coverage. One scheme is using a moving relay station mounted on the roof of the carriage. The mobile terminals are linked to base station via the relay station indirectly. This scheme has been detailed in $[4,12]$. The other scheme of realizing HSR communication is that the mobile terminals in the carriage are linked directly to the base stations on the ground. In this scheme, the electromagnetic waves suffer a penetration loss from the carriage windows [9]. The second scheme is adopted in this measurement.

\section{Analysis Method}

SAGE algorithm is proposed as a low-complexity approximation of the Maximum Likelihood (ML) estimation and successfully applied in different application purposes [13]. With this algorithm, accurate estimations of channel parameters from the measured data can be obtained. In the SAGE algorithm, the received and transmitted signals can be described as a finite number of plane waves [14]:

$$
r(t)=\sum_{l=1}^{L} \alpha_{l} e^{-j 2 \pi v_{l} t} s\left(t-\tau_{l}\right)
$$

where $L$ is the total number of extracted MPCs and $\alpha_{l}, \tau_{l}$, and $v_{l}$ are, respectively, the complex amplitude, delay, and Doppler frequency of the $l$ th MPC. The advantage of SAGE algorithm is that it can achieve the joint estimation of the complex amplification, delay, and Doppler frequency of the MPCs through iteration process. Based on the estimated value of delay and Doppler frequency, the LOS component is separated from the Non-Line of Sight (NLOS) components. At the second step with the achieved complex amplitude of each MPC, the path loss, shadow fading, and $K$-factor are calculated according to the definitions to give a description of the position-based fading characteristics of the HSR channel.

During the data analysis, the starting point of distance and time is chosen as the point of HSR train just passing by the base station with the 0 Doppler frequency shift. The T-R distance is proportional to time in the measurement.

\section{Measurement Results}

4.1. Path Loss. Path loss as a large-scale fading parameter is analyzed to help make a link budget for system design and base station site selection. To obtain the path loss, the traditional method usually averages snapshots corresponding to 5 40 wavelengths for removing the effects of small-scale fading. The SAGE algorithm has the ability to separate each MPC according to different delays, Doppler shifts, and amplitudes. With the method of just summing up the power of each MPC, the path loss can be calculated after removing the gain of antennas $(\mathrm{Tx} 17 \mathrm{dBi}, \mathrm{Rx} 7 \mathrm{dBi})$, penetration loss of carriage window $(12.4 \mathrm{~dB})$ and other influencing factors.

The statistical model is often expressed by

$$
P L(\mathrm{~dB})=10 \cdot n \cdot \log 10(d)+P L_{0}+X_{\sigma}(d),
$$

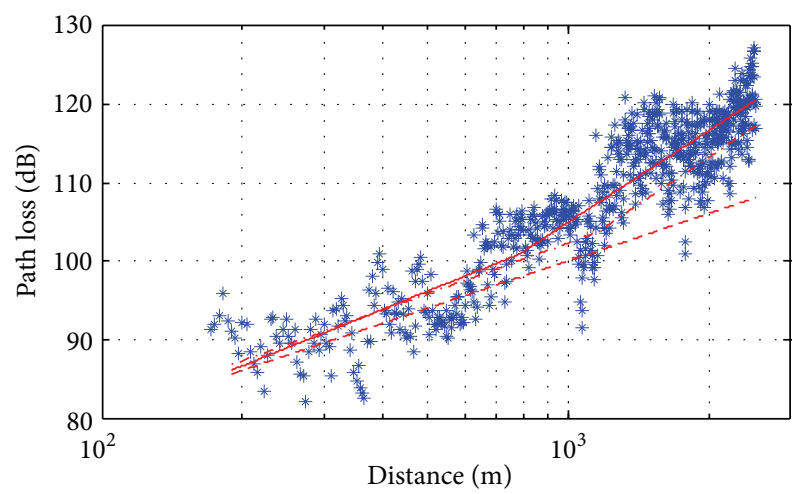

$$
\begin{array}{ll}
\text { * } & \text { Measured path loss } \\
\text { - - } & \text { Path loss fit } \\
\text { - - - Path loss model (WINNNER HSR scenario) }
\end{array}
$$

Figure 3: Path loss in hilly terrain.

where $n$ is the path loss exponent, $d$ is the distance between the transmitter and receiver, $P L_{0}$ is the intercept, and $X_{\sigma}(d)$ denotes the shadow fading with the standard deviation. Path loss depends on the distance between transmitter and receiver. Figure 3 shows the measured path loss and the fitting curve. It is noticed that there is a breakpoint within position-based measurement data. So the fitting broken curve is obtained by using the linear least square method in near and far regions relative to the base station, respectively. The breakpoint of the fitting curve is $788.6 \mathrm{~m}$ of T-R distance. At $788.6 \mathrm{~m}$, the value of path loss is $100.9 \mathrm{~dB}$. The equation of the path loss model with standard deviation $\sigma$ for this scenario is modeled by

$$
\begin{aligned}
P L & (\mathrm{~dB}) \\
& = \begin{cases}31.31+24.0 \log _{10}(d), & \sigma=3.3 \mathrm{~dB}, \quad d<788.6 \mathrm{~m}, \\
-11.6+38.8 \log _{10}(d), & \sigma=4.2 \mathrm{~dB}, \quad d \geq 788.6 \mathrm{~m} .\end{cases}
\end{aligned}
$$

The fitting curve can be regard as a two-slope model. For this model, the LOS path between transmitter and receiver is strong, and the contributions from all the scatters are small in a short distance. However, beyond the breakpoint distance, the scatters start to play an important role. In the two-slope model, the breakpoint distance [15] depending on the clearance of the first Fresnel zone is defined as

$$
r_{b}=4 \frac{h_{b} h_{m}}{\lambda}
$$

where $h_{b}$ and $h_{m}$ are the heights of the transmit antenna and receive antenna, respectively, and $\lambda$ is the wavelength. The $r_{b}$ is calculated as $1152 \mathrm{~m}$. This distance is assumed that there are no high obstacles around the propagation route. The measured distance $788.6 \mathrm{~m}$ is smaller than $r_{b}$. Our result is due to two fitting lines' intersection. This situation is attributed to certain scattering objects. The hills and huge buildings with similar height of the transmit antenna at the distance of $800 \mathrm{~m}-1000 \mathrm{~m}$ are all responsible for this 


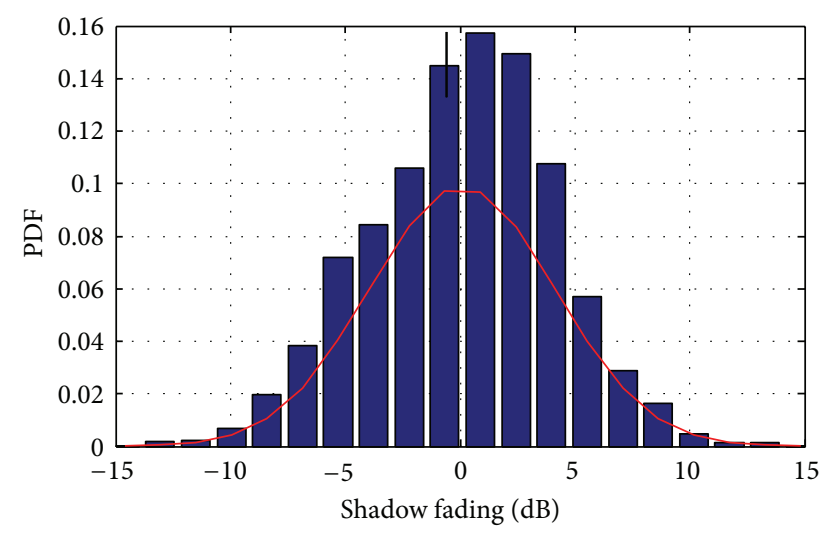

$\square$ Measured shadow fading

Normal fitting curve

FIGURE 4: PDF of shadow fading in hilly terrain.

phenomenon, where MPCs suffer complex constructive and destructive interference due to diffractions.

The free space path loss and the path loss model in the WINNER II HSR scenario [15] are also showed for comparison. The measured path loss suffers greater loss than the value in free space fading. The WINNER II path loss model is also described by the two-slope model with a breakpoint distance. From Figure 3, the fitting curve is very close to the WINNER II path loss model. The main differences exist not only in the aspect of breakpoint distance, but also in the aspect of slopes of the two segments. The slopes of the two segments of WINNER II path loss model are 21.5 and 40 , respectively. There is a visible gap between the slopes of WINNER II path loss model and the measured path loss in HSR hilly terrain scenario. The reason leading to the difference is that the scatters in hilly terrain scenario are richer than those in WINNER II HSR scenario. In short T$\mathrm{R}$ distance, these scattering waves have cancellation impact on the received signal energy. For long T-R distance, there is more scattered energy in this scenario than that in WINNER II HSR scenario.

4.2. Shadow Fading. Shadow fading is the variation of the path loss. After the path loss is obtained, shadow fading can be extracted from the calculated path loss after removing the distance-dependency fitting curve. This channel fading parameter is a variable caused by obstruction. It arises as slow fading, when the coherence time is large relative to the delay constraint of the channel. In most literatures, such as [15-17], the value of shadow fading is often regarded as a random variable conforming to the lognormal distribution. This is certified over a large number of measurement positions along the railway by Table 2 .

The comparison results of different distribution fitting methods are presented in Table 2. The Gamma distribution and the Nakagami- $m$ distribution are also used to model the shadow fading [16]. Compared with the other two distributions, the lognormal distribution fitting shows the
TABLE 2: Log likelihood value of different distribution fitting methods.

\begin{tabular}{lccc}
\hline & Lognormal & Gamma & Nakagami-m \\
\hline Log likelihood & -4343.36 & -4480.30 & -4919.28 \\
\hline
\end{tabular}

TABLE 3: Decorrelation distance statistic in hilly terrain.

\begin{tabular}{lcc}
\hline & Decorrelation distance $(\mathrm{m})$ & \\
\hline & $10 \%$ & 6.61 \\
Percentile & $50 \%$ & 10.28 \\
& $90 \%$ & 32.31 \\
& Mean & 14.81 \\
\hline
\end{tabular}

TABLE 4: $K$-factor statistic in hilly terrain.

\begin{tabular}{ccc}
\hline & $K$-factor $(\mathrm{dB})$ & \\
\hline & $10 \%$ & -9.05 \\
Percentile & $50 \%$ & -1.42 \\
& $90 \%$ & 4.51 \\
& mean & -1.85 \\
\hline
\end{tabular}

TABLE 5: The comparison of fading parameters in different scenarios.

\begin{tabular}{lcc}
\hline Scenarios & Viaduct [4] & Hilly terrain \\
\hline Frequency [GHz] & 2.35 & 2.4 \\
PL component & 3.03 & $2.40 / 3.88$ \\
PL intercept [dB] & 12.4 & $31.31 /-11.6$ \\
SF STD [dB] & 2.0 & $3.3 / 4.2$ \\
& Region $1:$ & Region $1:$ \\
K-factor [dB] & 23.05-0.0337d & $5.10-0.0102 d$ \\
& Region 2: & Region $2:$ \\
& $N(8.25,1.05)$ & $N(-1.85,5.05)$ \\
\hline
\end{tabular}

maximum log likelihood value. It is indicated that lognormal distribution fits the measured shadow fading well.

Figure 4 gives out the statistical characteristics of shadow fading in magnitude of $\mathrm{dB}$. In this figure, the blue bars are the probability distribution function, and the red curve is the normal fitting curve. The standard deviation of the fitting normal distribution is $3.99 \mathrm{~dB}$. Most values in this scenario range from $-10 \mathrm{~dB}$ to $10 \mathrm{~dB}$. The shadow fading in this scenario is mainly caused by the strong signal reflection from ground, hills, ponds, and buildings.

Shadow fading is often considered roughly constant over the symbol period. But in the HSR scenario, the rapid speed leads to the high Doppler frequency shift and small coherence time, which will influence the design of symbol period. Besides, the long T-R distance caused by rapid speed has a relationship with the change of shadow fading.

Figure 5 shows the relationship between the standard deviation of shadow fading in hilly terrain and the T-R distance. There are two turning points in the blue curve. One is around the position with the T-R distance of $650 \mathrm{~m}$. This position is between the water pond and the measured breakpoint with the distance of $788.6 \mathrm{~m}$. The distance of water pond from the base station is approximately $600 \mathrm{~m}$. 


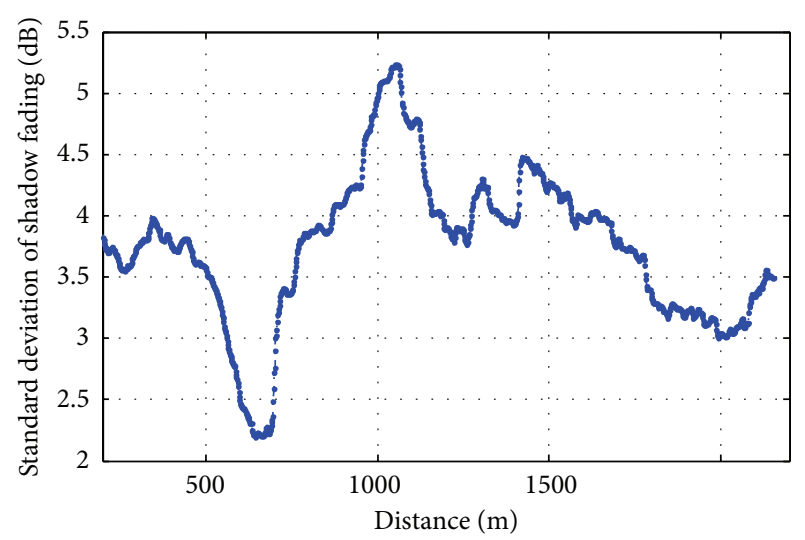

FIgURE 5: Standard deviation of shadow fading in hilly terrain.

The mirror refection from the water pond is highly powered, which makes the received energy in this region concentrated. So this leads to a deep drop in the curve with the position of $650 \mathrm{~m}$. But with the increasing distance, the opportunity for collecting the different scattering energy also increases. Consequently, the standard deviation of shadow fading is getting larger after this turning point. Another turning point is around the $1050 \mathrm{~m}$ similar to the calculated the breakpoint distance in a two-slope model. Beyond the last turning point, the energy of many MPCs is under the noise floor which is discarded in the data process. Then the standard deviation of shadow fading decreases with the increasing distance.

The autocorrelation of shadow fading is often investigated for power control and base station location designing. It is defined as the correlation of shadow fading in different positions under the same base station. The Spatial Channel Model (SCM) [18], 802.16J [19], and M.2135 [20] also give out the reference value and distance-dependent model of autocorrelation of shadow fading. The autocorrelation is described by the correlation coefficient in these documents. The definition is as follows:

$$
\rho_{1,2}=\frac{\mathrm{E}\left\{\mathbf{S}\left(d_{1}\right) \mathbf{S}\left(d_{2}\right)\right\}}{\sigma\left(d_{1}\right) \sigma\left(d_{2}\right)},
$$

where $\mathbf{S}\left(d_{1}\right)$ and $\mathbf{S}\left(d_{2}\right)$ are the values of shadow fading in the position with distance $d_{1}$ and $d_{2}$ and $\sigma\left(d_{1}\right)$ and $\sigma\left(d_{2}\right)$ are the standard deviations of shadow fading $\mathbf{S}\left(d_{1}\right)$ and $\mathbf{S}\left(d_{2}\right)$, respectively.

Figure 6 shows the autocorrelation coefficient of the shadow fading between the position with T-R distance of $200 \mathrm{~m}$ and the positions behind. When the autocorrelation coefficient is less than 0.5 , the decorrelation distance $d_{\text {cor }}$ is determined. In Figure 6, the decorrelation distance is $10.28 \mathrm{~m}$. This value is smaller than the value of $20 \mathrm{~m}$ suggested in 802.16 J for vehicular test environment and is also smaller than the value of about $40 \mathrm{~m}$ for LOS scenario of RMa in M. 2135. The red dotted line is on the basis of 802.16J model with

$$
\rho(\Delta d)=e^{-\left(|\Delta d| / d_{\text {cor }}\right) \ln 2},
$$

where $\Delta d$ is the distance between the two observed positions. It seems that there is a little difference between the measured

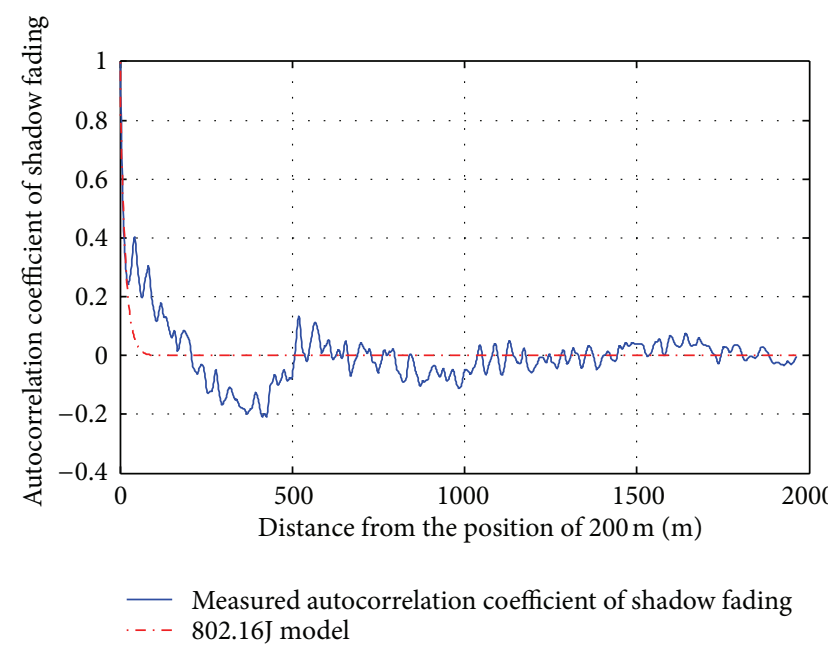

FIGURE 6: Autocorrelation coefficient of shadow fading in hilly terrain.

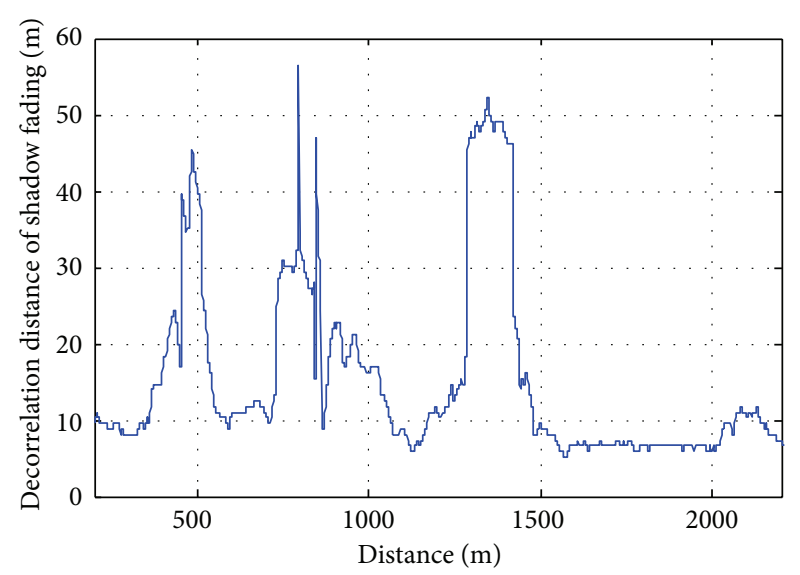

FIGURE 7: Decorrelation distance of shadow fading in hilly terrain.

autocorrelation coefficient and the published model, due to large fluctuations with $\Delta d$ which is from $100 \mathrm{~m}$ to $400 \mathrm{~m}$. These fluctuations are caused by the wave reflection from ground and other scattering objects.

Figure 7 depicts the decorrelation distance of all the positions in the whole route. Each value of decorrelation distance is calculated between the certain position and other positions with the range of $200 \mathrm{~m}$ behind. In most cases, the value of decorrelation distance is around $10 \mathrm{~m}$. Due to the observable LOS, the effects of scatters in these locations are small. There are also many positions with decorrelation distance of more than $30 \mathrm{~m}$ which is similar to the correlation distance for LOS scenario of RMa in M. 2135. The phenomenon reflects the nonstationary characteristics of large-scale fading parameters. This is the difference between the HSR hilly terrain scenario and custom outdoor scenarios. These positions with larger decorrelation distance often appear before, around, or behind the positions of huge scattering objects, such as the water pond with the distance of $600 \mathrm{~m}$ and the huge building with the distance of $1400 \mathrm{~m}$. It is verified that the 
reflected waves received by different positions from the same scattering object experience the similar fading, when these receiving positions are at the same direction relative to the same scattering object.

Table 3 shows the statistical results of decorrelation distance. The mean value of decorrelation distance is $14.81 \mathrm{~m}$. In the measured route, $90 \%$ of all locations exhibit decorrelation distance less than $32.31 \mathrm{~m}$, and $50 \%$ of all positions exhibit decorrelation distance less than $10.28 \mathrm{~m}$. But there are also an amount of positions with a larger decorrelation distance of more than $30 \mathrm{~m}$ which exceeds $10 \%$. Therefore, most of the decorrelation distance values are around $10.28 \mathrm{~m}$, but the phenomenon that there is a great difference of the decorrelation distance in different positions is not ignored. The propagation environment with large scattering objects needs special attention for communication system design.

4.3. K-Factor. Fast fading represents a rapid amplitude variation of received signal for movements in the order of a wavelength [21]. For LOS scenario, $K$-factor is an important parameter for describing the fast fading characteristic of the channel. It is used for describing the ratio of the power of dominant LOS component to the power of Rayleigh NLOS components. The traditional definition is as follows:

$$
K(\mathrm{~dB})=10 \log _{10} \frac{\rho^{2}}{\sigma^{2}},
$$

where $\rho^{2}$ is the power of LOS component and $\sigma^{2}$ is the total power of NLOS components [22]. It is assumed that the LOS component is the first arrival of MPCs with the smallest delay. Therefore, these two parameters $\rho^{2}$ and $\sigma^{2}$ can be easily calculated with the amplitudes of extracted MPCs in SAGE algorithm. According to the definition, the broadband $K$ factor is achieved.

Since $K$-factor is related to the probability of a fade of certain depth, it is critical for improving the communication quality. In traditional view, the high-speed problem can be solved in some degree either through increasing the SNR by $2 \mathrm{~dB}$ or through increasing the $K$-factor by $6 \mathrm{~dB}$ [23]. For HSR scenario, the relationship between the $K$-factor and the T-R distance is worth investigating for system designing.

The relationship between the $K$-factor and the T-R distance is shown in Figure 8. It is obvious that there are also 2 segments of measured $K$-factor. The fitting curve is obtained by using the linear least square method in near and far regions. In this figure, the breakpoint of fitting curve might be set as the calculated breakpoint with the T-R distance of $1152 \mathrm{~m}$ which is mentioned in the two-slope model of Section 4.1 as well. The expression of the red fitting curve is as follows:

$$
K(\mathrm{~dB})= \begin{cases}5.10-0.0102 d, & d<1152 \mathrm{~m}, \\ -1.19-0.0004 d, & d \geq 1152 \mathrm{~m} .\end{cases}
$$

In the near region, the phenomenon that median of $\mathrm{K}$-factor decreases linearly with increasing T-R distance is verified as depicted in [21]. The traditional explanation is that the signal power decay is logarithmic with distance, and the

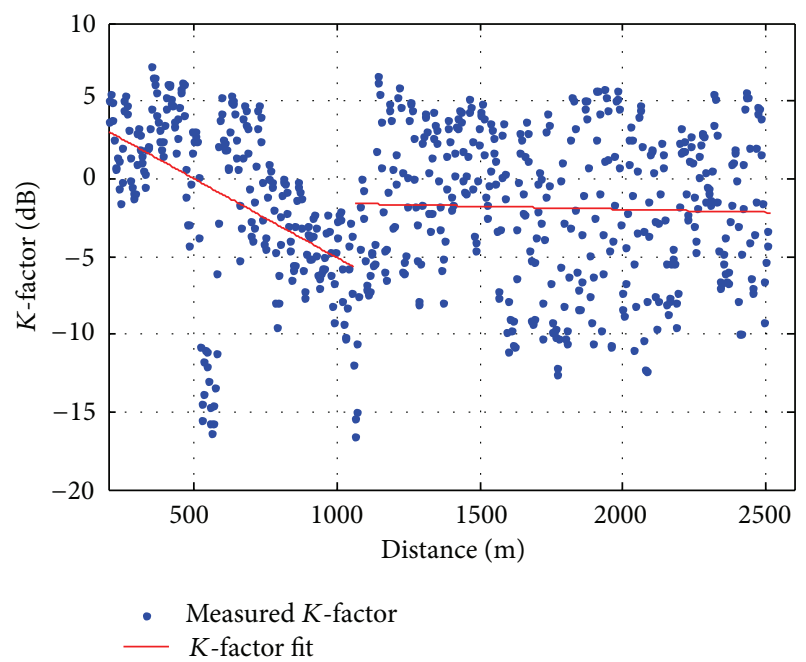

Figure 8: $K$-factor in hilly terrain.

decreasing power of multipath components is much smaller than the decreasing power of LOS one when the distance increases. The standard deviation between the measured $K$ factor and fitting curve is approximately limited to $3 \mathrm{~dB}$. In the far region, the values of the red fitting curve lie between -1.5 and $-2.5 \mathrm{~dB}$. It is indicated that $K$-factor suffers fluctuation which is often estimated as a Gaussian variable [23] with the mean value of $-1.85 \mathrm{~dB}$ and the standard deviation of $5.05 \mathrm{~dB}$. The $K$-factor value for $10 \%, 50 \%$, and $90 \%$ points of the cumulative distribution function (CDF) of the Ricean $K$ factor in far region can be found in Table 4. From Table 4, the $K$-factor values of $50 \%$ and $90 \%$ positions are lower than $-1.42 \mathrm{~dB}$ and $4.51 \mathrm{~dB}$, respectively. The mean $K$-factor value of all positions is $-1.85 \mathrm{~dB}$. Most of $K$-factor values are near $0 \mathrm{~dB}$. There are also $10 \%$ of positions in far region with a $K$ factor less than $-9 \mathrm{~dB}$. This means that the power of LOS path remains at a low level in the measured route. Actually in some cases, the LOS signal is not the strongest signal. These cases include the impact of the main scattering objects and the long distance transmission. The results are statistically significant.

This result of $K$-factor is different from the WINNER II HSR result of measured $K$-factor. In WINNER II HSR result, the $K$-factor is constant over the measured distance. As Figure 8 indicates, the $K$-factor has its own special characteristic in different positions of the hilly terrain scenario. For example, the water pond which caused the strong specular reflection in the T-R distance of about $600 \mathrm{~m}$ is mentioned once again. The $K$-factor has an extreme low value.

4.4. Comparison with Viaduct Scenario. Viaduct is another typical scenario in HSR scenarios. Table 5 compares the results of the channel fading parameters in viaduct scenario and the results in hilly terrain scenario. The viaduct measurement is conducted in the Beijing-Tianjin HSR by the Beijing Jiaotong University with an EB CS radio channel sounder Propsound at a frequency of $2.35 \mathrm{GHz}$ with $10 \mathrm{MHz}$ band 
width [4]. The second is the measured hilly terrain scenario in this paper.

Although differences exist in measurement frequency and other aspects, the comparison between two measurements is still meaningful. In Table 5, the distinction of channel parameters between two scenarios is obvious. Compared with the viaduct scenario, the measured path loss in hilly terrain is divided into two parts with a breakpoint. The path loss component of the first region in hilly terrain is smaller than the value in viaduct, but the value of second region in hilly terrain is larger than that in viaduct. Due to the rapidly changing propagation environment along the measured route, the standard deviation of shadow fading in hilly terrain is larger than that in viaduct. The rich scattering environment of hilly terrain also leads to smaller $K$-factor, compared with viaduct. The difference of fading characteristics between viaduct and hilly terrain indicates that the work on HSR hilly terrain measurement and modeling is necessary for general applicability of HSR.

\section{Conclusions}

Through the use of the high-resolution SAGE algorithm in HSR hilly terrain scenario, the statistical fading characteristic of wireless channel parameters has been analyzed in this paper. The HSR hilly terrain scenario is characterized by an obvious LOS component, complex scattering propagation environment, and rapid change of propagation condition due to high speed. On the whole, our measurement results are supported by the fact that the values of fading parameters are changing with time or T-R distance.

In the analysis, the path loss, shadow fading, and $K$ factor are investigated. Compared with the free space path loss model, the measured path loss value in hilly terrain scenario suffers greater decay and shows the property of having a breakpoint distance as the WINNER HSR model shows. The shadow fading is a parameter for describing the slow fading characteristic. It is lognormal fitting and changed with two turning points. As an important point of interest, the autocorrelation of shadow fading is investigated. It is exhibited that the mean value of decorrelation distance is around $14 \mathrm{~m}$. But the value will rise up when the train gets near to the huge reflectors. The $K$-factor as a fast fading parameter is also influenced by the breakpoint distance. Within the breakpoint distance, $K$-factor decreases as the T-R distance increases. Beyond the break point distance, the value of $K$-factor fluctuates dramatically. These results are compared with the published results in viaduct scenario, and the difference between viaduct and hilly terrain is summarized. The results of this paper can be utilized to build a positionbased channel fading model and amend the existing channel models for HSR hilly terrain scenario. And this is also hoped to serve as a reference for future HSR communication system design, evaluation, and improvement.

\section{Acknowledgments}

This work has been partially supported by the National Basic Research Program of China (2012CB316002), National
Natural Science Foundation of China (61201192), Tsinghua University Initiative Scientific Research Program (2011081025), National S\&T Major Project (2013ZX03001024), International S\&T Cooperation Program (2012DFG12010), Tsinghua-Qualcomm Joint Research Program, China Postdoctoral Science Foundation, and Open Research Fund of National Mobile Communication Research Laboratory, Southeast University. The authors also would like to thank Mr. Zhenghui Li, Wenzhen Pang, and Yuejian Zhou for their help in the basic work of channel measurement.

\section{References}

[1] http://en.wikipedia.org/wiki/High-speed_rail.

[2] P. Lukasazewics and E. Anderson, "Green train energy consumption, estimation on high-speed rail operation," Tech. Rep. 978-91-7415-257-9, KTH Railway Group, Stockholm, Sweden, 2009.

[3] J. Li and M. Kavehrad, "Effects of time selective multipath fading on OFDM systems for broadband mobile applications," IEEE Communications Letters, vol. 3, no. 12, pp. 332-334, 1999.

[4] L. Liu, C. Tao, J. Qiu et al., "Position-based modeling for wireless channel on high-speed railway under a viaduct at $2.35 \mathrm{GHz}$," IEEE Journal on Selected Areas in Communications, vol. 30, pp. 834-845, 2012.

[5] P. Aikio, R. Gruber, and P. Vainikainen, "Wideband radio channel measurements for train tunnels," in Proceedings of the 48th IEEE Vehicular Technology Conference (VTC '98), pp. 460464, Ottawa, Ontario, Canada, May 1998.

[6] D. J. Cichon, T. Zwick, and W. Wiesbeck, "Ray optical modeling of wireless communications in high-speed railway tunnels," in Proceedings of the IEEE 46th Vehicular Technology Conference (VTC '96), pp. 546-550, Atlanta, Ga, USA, May 1996.

[7] L. Gao, Z. Zhong, B. Ai, and L. Xiong, "Estimation of the Ricean K factor in the high speed railway scenarios," in Proceedings of the 5th International ICST Conference on Communications and Networking in China (ChinaCom '10), Beijing, China, August 2010.

[8] J. Qiu, C. Tao, L. Liu, and Z. Tan, "Broadband channel measurement for the high-speed railway based on WCDMA," in Proceedings of the IEEE 75th Vehicular Technology Conference (VTC '12-Spring), pp. 1-5, Yokohama, Japan, May 2012.

[9] A. Ghazal, C. X. Wang, H. Haas, M. A. Beach, X. Lu, and D. Yuan, "A non-stationary MIMO channel model for high speed train communication systems," in Proceedings of the IEEE 75th Vehicular Technology Conference (VTC '12-Spring), pp. 347-351, Yokohma, Japan, May 2012.

[10] Google Maps, http://maps.google.com.hk/.

[11] Y. Rui, Y. Zhang, S. Liu, and S. Zhou, " $3.52 \mathrm{GHz}$ MIMO radio channel sounder," in Proceedings of the International Conference on Communications, Circuits and Systems (ICCCAS '08), pp. 7983, May 2008.

[12] WINNER2 WP1, "Measurement results and analysis items," Internal Report IR1.1.230.9.2006, 2006.

[13] B. H. Fleury, M. Tschudin, R. Heddergott, D. Dahlhaus, and K. I. Pedersen, "Channel parameter estimation in mobile radio environments using the SAGE algorithm," IEEE Journal on Selected Areas in Communications, vol. 17, no. 3, pp. 434-450, 1999.

[14] S. Wyne, A. F. Molisch, P. Almers, G. Eriksson, J. Karedal, and F. Tufvesson, "Outdoor-to-indoor office MIMO measurements 
and analysis at $5.2 \mathrm{GHz}$," IEEE Transactions on Vehicular Technology, vol. 57, no. 3, pp. 1374-1386, 2008.

[15] WINNER2 WP1, "Radio channel measurement and analysis results," Deliverable D 1.1.2, ver 1.0. 30.9.2007, 2007.

[16] J. Salo, L. Vuokko, and P. Vainikainen, "Why is shadow fading lognormal?" in Proceedings of the International Symposium on Wireless Personal Multimedia Communications, pp. 522-526, Aalborg, Denmark, September 2005.

[17] I. Forkel, M. Schinnenburg, and M. Ang, "Generation of twodimmensional correlated shadowing for mobile radio network simulation," in Proceedings of the Wireless Personal Multimedia Communications (WPMC '04), pp. 314-319, Abano Terme, Italy, September 2004.

[18] 3GPP TR 25.996, "3rd Generation Partnership Project; technical specification group radio access networks; spatial channel model for MIMO simulations (release 6)," V6.1.0.

[19] "Multi-hop relay systemevaluation methodology (Channel model and performance metrics)," IEEE802.16j-06/013r3, 2007.

[20] ITU-R, "Guidelines for evaluation of radio interface technologies for IMT-Advanced," Tech. Rep. ITU-R M.2135, 2008.

[21] Z. Wang, E. K. Tameh, and A. R. Nix, "Statistical peer-to-peer channel models for outdoor urban environments at $2 \mathrm{GHz}$ and $5 \mathrm{GHz}$," in Proceedings of the IEEE 60th Vehicular Technology Conference (VTC '04-Fall), pp. 5101-5105, September 2004.

[22] W. H. Jeong, J. S. Kim, M. Jung, and K. Kim, "MIMO channel measurement and analysis for $4 \mathrm{G}$ mobile communication," Computer Science, vol. 7425, pp. 678-682, 2012.

[23] M. Goller, "Application of GSM in high speed trains: measurement and simulations," IEEE Colloquium on Radio communications in Transportation, pp. 5/1-5/7, 1995. 

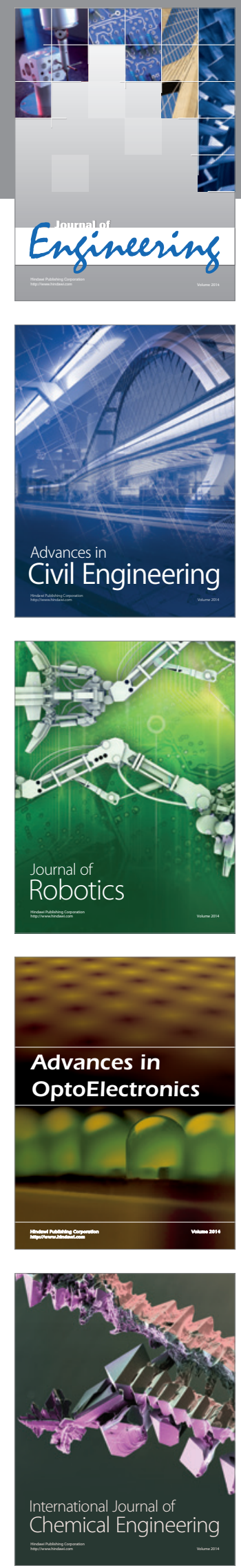

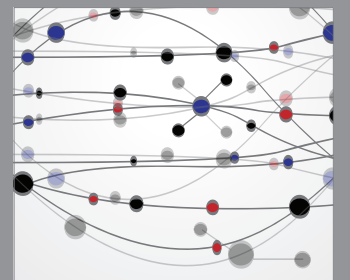

The Scientific World Journal
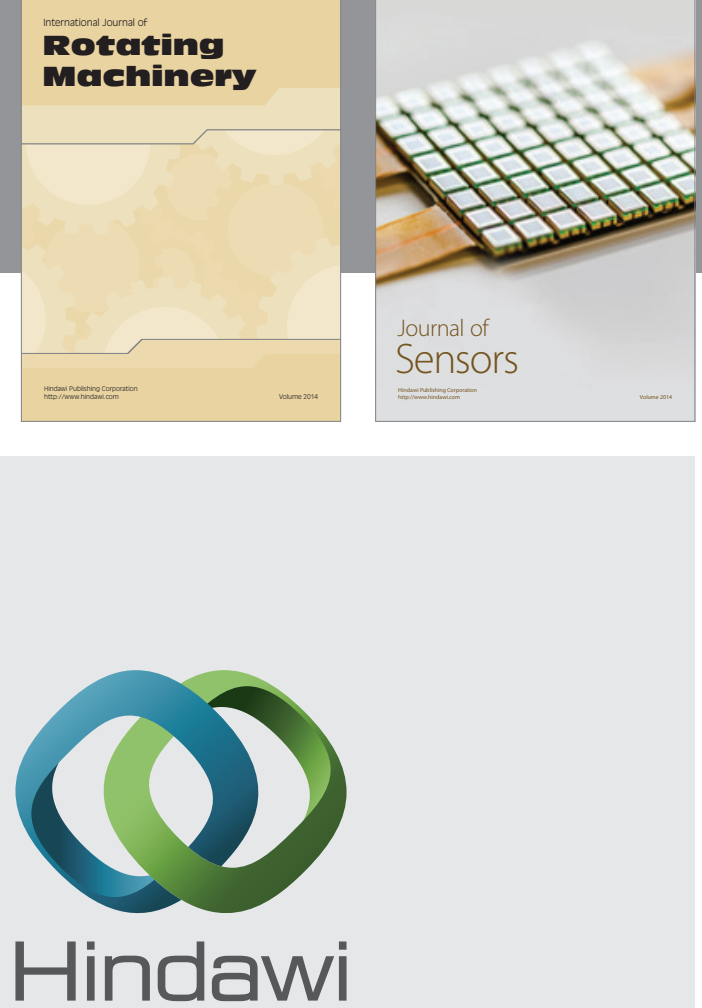

Submit your manuscripts at http://www.hindawi.com
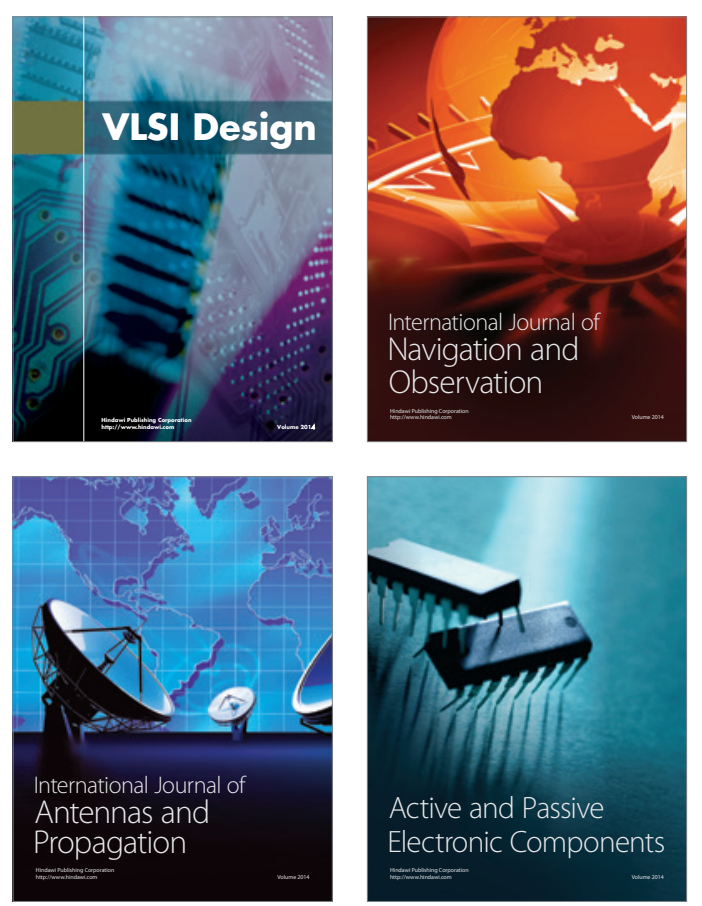
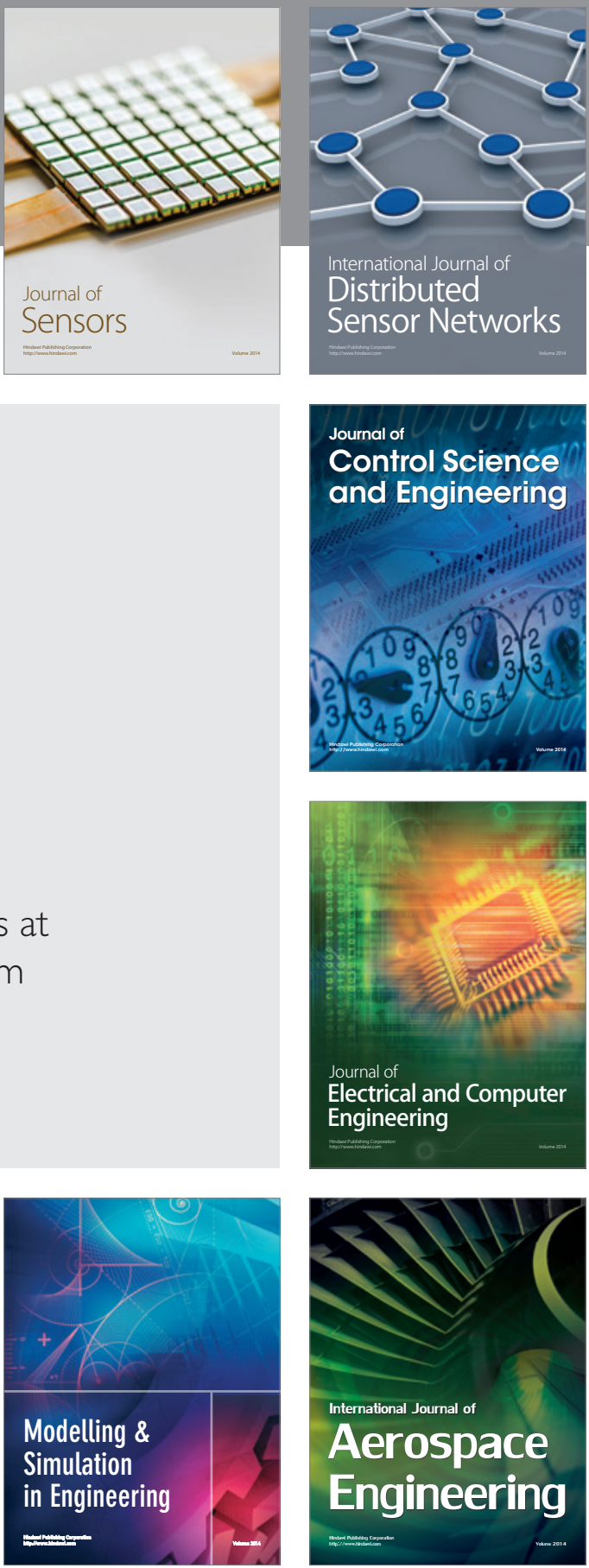

Journal of

Control Science

and Engineering
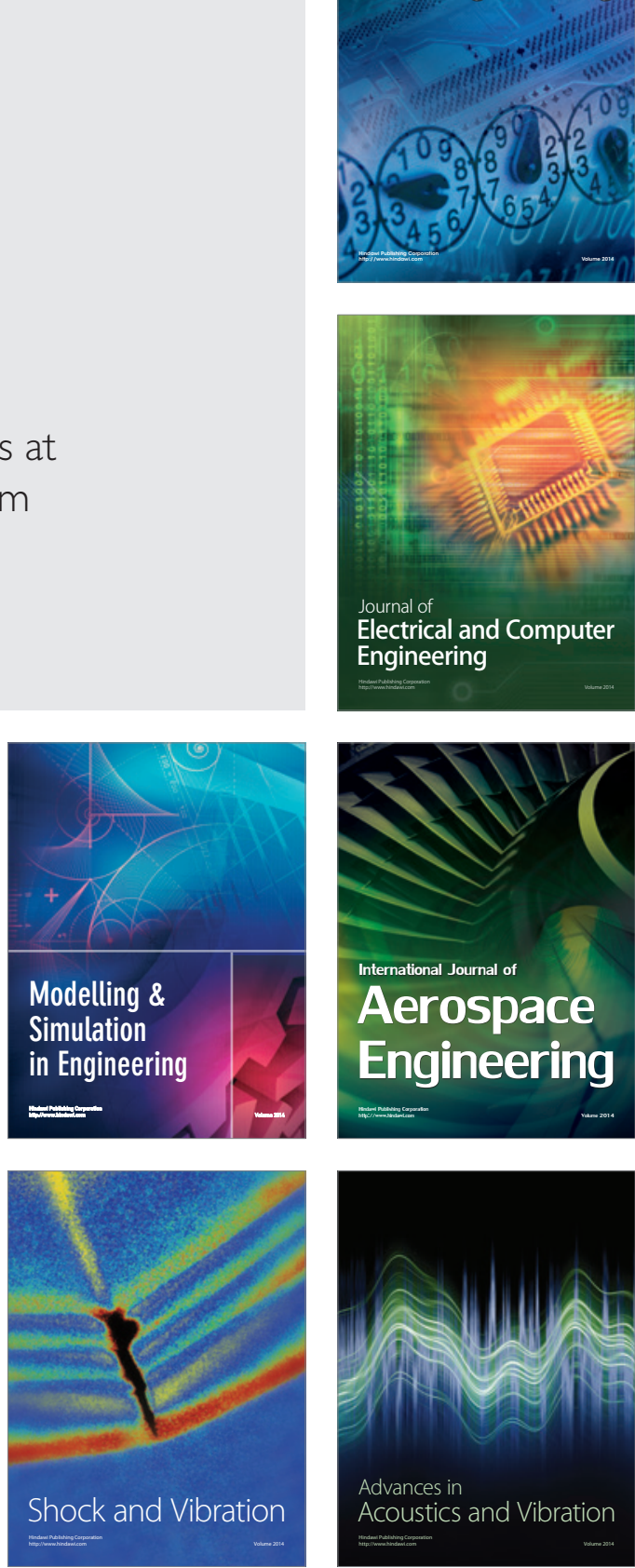